\title{
Transferencia de aprendizajes desde el aula formal hacia la práctica clínica: una reflexión sobre la formación de fonoaudiólogos en Chile
}

\author{
Transferring learning from the formal classroom to \\ the clinical practice: A reflection on the training of \\ speech therapists in Chile
}

\section{Felipe Torres}

Departamento de Fonoaudiología

Universidad de Chile

Carlos Álvarez

Departamento de Fonoaudiología

Universidad de Chile

Luis Romero

Departamento de Fonoaudiología Universidad de Chile
Contacto con el autor: Felipe Torres M. Santiago - Chile Correo-e: felipetorres@med.uchile.cl

\section{RESUMEN}

En el presente artículo se reflexiona sobre el proceso de transferencia de los aprendizajes desde el aula formal hacia la práctica clínica y sobre el desafío que implica esta tarea en la formación de fonoaudiólogos en instituciones de educación superior chilenas. Para ello, se inicia con un análisis sobre los tres niveles del sistema educacional actual en Chile, el macrosistema (modelo educativo general), mesosistema (modelo educativo asumido por cada una de las instituciones de educación superior) y microsistema (currículo adoptado por cada carrera universitaria), con el fin de entregar un marco referencial. Posteriormente, se describe el Modelo de Enseñanza para la Comprensión, se plantea evidencia de aplicación en instituciones de educación superior de Latinoamérica y se reflexiona sobre el aporte que significaría la implementación de este modelo para el proceso de transferencia de los aprendizajes. Por último, se plantean los alcances de esta propuesta y las posibles limitaciones en su ejecución.

Palabras clave: enseñanza para la comprensión, educación universitaria, conocimientos, transferencia de aprendizajes, constructivismo, metacognición.

\section{ABSTRACT}

In this paper, we present some reflections on the process of learning-transfer from formal classrooms to clinical practices, and the challenges involved for the training of speech therapist in Chilean higher-education institutions. We begin by analyzing the three levels of the current educational system in Chile: the macrosystem (general educational model), the mesosystem (educational models assumed by each higher-education institution), and microsystem (curricula adopted at universities), in order to provide a reference framework. Subsequently, we describe the Teaching Model for Understanding, we present evidence of application in institutions of higher-education in Latin America and we reflect on the impact that the implementation of this model might have for the process of transfer of learning. Finally, we consider the scope of the proposal and the possible limitations in its execution.

Keywords: teaching for understanding, tertiary education, knowledge, knowledge transfer, constructivism, metacognition.

Recibido: $11 / 09 / 2017$ Aceptado: 01/08/2018 


\section{Introducción}

El aprendizaje, considerado como una de las actividades más importantes en la vida del ser humano (Pastorino \& Doyle-Portillo, 2016), es un fenómeno dinámico de origen multidimensional, que resulta de procesos experienciales interactivos que tiene un organismo en un contexto determinado (Lefrancois, 2012; Lund, 2010). Dichas experiencias interactivas, que involucran procesos cognitivos y asociativos, producen cambios relativamente permanentes en el comportamiento potencial de un organismo, entre estos, el ser humano (Klein, 2012). El aprendizaje, en su origen multidimensional, es definido por Wray \& Lewis (1997) como un proceso social, situado y metacognitivo, en el que interactúan el conocimiento previo con aquel que será aprendido. Estos principios fundamentales establecen que el ser humano no es un organismo pasivo en este proceso. Por el contrario, se ha determinado que una de las dimensiones que posibilitan el aprendizaje es el rol activo que ejercen los aprendientes en el proceso de construcción del propio conocimiento (Resnick, 1999; Resnick \& Collins, 1996). En este sentido, el aprendizaje es entendido como un proceso social interactivo y no como una dinámica unidireccional de transmisión de conocimiento desde quien lo posee, en este caso, el profesor, hacia el aprendiente (Van Berkel, Scherpbier, Hillen, \& van der Vleuten, 2010). Por tanto, la práctica pedagógica en todos los niveles del sistema educacional debería propender, de manera permanente, al establecimiento de condiciones que potencien el protagonismo del estudiante para el logro de un aprendizaje significativo (Gaete-
Quezada, 2011).

Nuestra experiencia académica en programas chilenos de pregrado en Fonoaudiología, nos muestra que la práctica pedagógica se ha orientado tradicionalmente, a la transmisión de conocimientos por parte del profesor a los estudiantes y a la búsqueda del desarrollo de habilidades clínicas procedimentales en estos últimos, para su futuro desempeño profesional. Estos dos aspectos, suelen estar integrados tanto en el trabajo de aula que se realiza en la mayoría de los cursos de los planes curriculares propios de cada institución, como en el trabajo realizado en contextos clínicos. Sin embargo, pese a esta integración, resulta frecuente observar una disociación entre el desempeño de los estudiantes en su trabajo teórico-práctico de aula y su posterior desempeño en su trabajo extra-aula, a saber, en un contexto clínico. Consiguientemente, es posible establecer que un buen rendimiento en el aula no siempre está relacionado con un buen rendimiento en el trabajo práctico llevado cabo durante los últimos años de la formación profesional. Esto se debe a que la aplicación en contextos prácticos de los saberes desarrollados en el trabajo de aula resulta ser un proceso con complejidades propias que no siempre son abordadas en la formación académica de los futuros fonoaudiólogos. Asumiendo que la disociación antes mencionada puede estar relacionada, entre otras cosas, con la falta de trabajo de aula orientado a desarrollar procesos que permitan el logro de niveles comprensivos significativos por parte de los estudiantes, se analiza la problemática desde el modelo Enseñanza para la Comprensión (Teaching for Understanding), propuesto por la organización 
Project Zero, perteneciente a la Harvard Graduate School of Education, Harvard University ${ }^{1}$.

En este modelo, se propone que el objetivo principal del proceso educativo debe estar orientado a que los estudiantes logren comprender fenómenos de variada naturaleza con los que los seres humanos convivimos a diario. Desde este paradigma, se entiende por comprensión a la acción de pensar y actuar con flexibilidad a partir de lo que se sabe, es decir, que la comprensión es la acción de pensar en el conocimiento (Salgado-García, 2012; Stone, 1999). En este sentido, se establece que un fenómeno no es comprendido por el solo hecho de poseer conocimiento o haber desarrollado una habilidad respecto de este (Perkins, 1993), sino que la comprensión de un fenómeno se logra cuando es posible explicarlo, interpretarlo y aplicar sus principios a la resolución de problemas que surgen en nuevas situaciones (McTighe \& Self, 2014). Debido a lo anterior, se propone que al aplicar la enseñanza para la comprensión en la formación de fonoaudiólogos podría facilitar la transferencia de aprendizajes desde el aula a los contextos prácticos, entendiendo que, en estos, el estudiante debe explicar, interpretar y aplicar conocimientos en situaciones clínicas siempre nuevas (Safran \& Muran, 2005).

\footnotetext{
${ }^{1}$ El Proyecto Zero fue fundado por el filósofo Nelson Goodman en la Escuela de Postgrado de Educación en Harvard, en el año 1967, con el objetivo de estudiar y mejorar la educación en las artes. Desde ese momento hasta la actualidad, el Proyecto Zero sigue funcionando en la Universidad de Harvard y ha aportado a los procesos educativos de distintos países del mundo.
}

\section{Niveles del sistema educacional en Chile}

Como marco referencial a la problemática, se realiza un análisis sobre los tres niveles del sistema educativo actual en Chile, por una parte, el macrosistema (modelo educativo general), mesosistema (modelo educativo asumido por cada una de las instituciones de educación superior) y microsistema (currículo adoptado por cada carrera universitaria). En relación con el modelo educativo actual (macrosistema), la Ley General de la Educación (LGE) que rige la enseñanza en todos los niveles educativos chilenos, propone un sistema basado en la estandarización. Espinola \& Claro (2010) plantean que una reforma basada en estándares busca explicitar las metas que deben alcanzar los actores educacionales, contar con mecanismos que permitan evaluar estos estándares y tomar decisiones en consideración de los resultados. Para Espinola \& Claro (2010) y Casassus (2010), este modelo desvaloriza aspectos humanistas, emocionales y de convivencia social, generando una visión simplificada del proceso educativo. Así mismo, plantea que un modelo de esta naturaleza ejerce un proceso reduccionista del proceso educativo: se enseña solo con objeto de alcanzar los estándares establecidos. Finalmente, en su crítica al sistema educativo, este autor menciona que el método de premios y castigos asociados a los resultados reduce la calidad de las interacciones profesor-estudiante. No cabe duda de que el objetivo principal de una institución de educación superior es que sus estudiantes logren los estándares académico-profesionales establecidos. Sin embargo, 
la búsqueda de dicho logro tiende a convertir el proceso de enseñanza en un entrenamiento específico para el proceso de evaluación. Este hecho puede explicar la razón por la cual las sesiones de clases, en alguna medida, continúan inmersas en una dinámica de traspaso unidireccional de conocimientos del docente al estudiante, a pesar de que Chile ha transitado desde un modelo centrado en la enseñanza a otro centrado en el aprendizaje, donde el énfasis esta puesto en el proceso que el estudiante debe realizar para aprender y no en el docente ni en la información que este debe entregar. Este es un factor que promueve la práctica rutinaria de las habilidades básicas y la estandarización de la enseñanza (Resnick, 1999). Todo lo anterior, dificulta, en gran medida, el proceso comprensivo que un estudiante debería lograr en su proceso de formación académico-profesional. Dicho logro formativo requiere de esfuerzos de todos los actores de la comunidad educativa en su conjunto y requiere de un modelo que promueva en los estudiantes el desarrollo de procesos que le permitan observar fenómenos de manera crítica, como resultado de procesos de razonamiento explicativo, interpretativo y resolutivo.

Por otra parte, con respecto al mesosistema (modelo educativo asumido por cada una de las instituciones de educación superior) y microsistema (currículo adoptado por cada carrera universitaria), es indispensable que ambos se encuentren en sintonía en todo momento durante la ejecución de un programa educacional en una determinada institución. En años recientes, varias instituciones de educación superior chilenas han declarado un proceso de innovación del currículo de pregrado.
Esta innovación se materializa mediante la transición de un currículo centrado en asignaturas y contenidos, a un currículo basado en competencias (Martínez et al., 2006).

El proceso de innovación curricular mencionado está vinculado a la iniciativa Alpha-Tuning (González \& Wagenaar, 2003). Esta propuesta europea promueve cambios relacionados con las transformaciones de la sociedad y los avances tecnológicos, así como el concepto de globalización, pensando en un mundo cada vez más conectado y con mucho intercambio de conocimientos. Así, se inician los procesos de innovación curricular bajo el paradigma del currículum orientado en competencias, lo que ha significado un cambio radical respecto de la mirada tradicional (Hawes et al., 2017; Troncoso \& Hawes, 2007).

La innovación curricular basada en competencias se cuestiona sobre cómo se construye el conocimiento. En este sentido, se discute respecto del aprendizaje "teórico" y "práctico" y de cómo estos estarían desintegrados en un currículo tradicional. Una de las mayores críticas a los planes curriculares tradicionales es su diseño a partir de unidades de conocimiento más bien independientes centradas en el docente y su saber (Perrenoud, 2004). Por tanto, el conocimiento construido tendría la dificultad de que es el estudiante (futuro profesional) quien debe integrarlo en instancias específicas.

El nuevo currículo que se propone tiene un fuerte énfasis en la integración de conocimientos y entre los factores que permitirían dicha integración corresponde a la noción de competencia, la cual se concibe como "saber actuar" (Jonnaert, Masciotra, 
Barrette, Morel, \& Mane, 2007; Le Boterf, 2004; Perrenoud, 2004). Es en este contexto que el estudiante de Fonoaudiología experimenta el tránsito por un currículo que lo expone a instancias en donde debe movilizar el conocimiento teórico y práctico con objeto de resolver problemas siempre nuevos.

A pesar de los avances que significa pasar de un currículo centrado en asignaturas y contenidos, a uno centrado en competencias, este último ha recibido críticas dado que no explicita ni asegura el desarrollo de pensamiento crítico, reflexivo ni metacognitivo en los estudiantes. Este desarrollo es un proceso necesario para lograr la transferencia desde conocimientos adquiridos en aula hacia la aplicación en campos clínicos (Díaz, 2006; Rodríguez, 2007).

\section{Modelo de Enseñanza para la Comprensión}

Este modelo, desarrollado en la Escuela de Postgrado de la Universidad de Harvard, propende en los docentes la posibilidad de reflexión acerca de su propia práctica pedagógica y su resignificación. En los estudiantes, este modelo promueve el proceso de reflexión acerca de las diversas temáticas que están aprendiendo. Además, promueve en ellos el establecimiento de relaciones entre variados aspectos de la vida diaria y los contenidos tratados en el curso, entre los principios teóricos y la práctica, entre el pasado y el presente, entre el presente y el futuro (Blythe, 1999; Perrenoud, 2007).

El modelo de enseñanza para la comprensión entiende el proceso de enseñanza-aprendizaje lejos de la mera transferencia y recepción de información, situándolo, por el contrario, en un plano de desarrollo social, natural, activo, integrado y contextualizado, centrado en el estudiante (Jubert, Pogliani, Tocci, \& Vallejo, 2011).

Bajo este modelo, se propone la implementación de prácticas pedagógicas de aula considerando cuatro pilares fundamentales. El primer pilar, tópicos generativos, establece que el núcleo del currículo debe estar formado por un tema que sea central para la disciplina, que sea interesante para el profesor, accesible para los estudiantes y que sea fácil de vincular con las experiencias de estos últimos. El segundo pilar, metas de comprensión, establece que es indispensable la definición tanto de metas específicas de comprensión para cada tópico como metas abarcadoras. Ambas metas generan el mapa de progreso del proceso. El tercer pilar, desempeños de comprensión, promueve la consideración de los sucesivos desempeños de los estudiantes para el establecimiento de nuevos desafíos con aumentos sutiles de dificultad. Por último, el cuarto pilar, evaluación diagnóstica continua, hace referencia a que la evaluación debe ser un proceso permanente, con criterios públicamente explicitados, con constante retroalimentación y reflexión por parte de los estudiantes, ya que está orientado al aprendizaje (Blythe, 1999).

Por lo tanto, la Enseñanza para la Comprensión requiere docentes que promuevan en el estudiante procesos de formulación de inferencias, hipótesis y el establecimiento de soluciones a situaciones conflictivas en contextos generales del diario vivir y en contextos más específicos en los cuales se ejerce 
profesionalmente. Así mismo, requiere de la planificación de actividades que busquen estimular procesos de comparación, búsqueda de patrones, generalización y automonitoreo del proceso de enseñanza-aprendizaje en los estudiantes (SalgadoGarcía, 2012).

Dado lo anterior, la promoción del desarrollo de la comprensión no solo tendría un efecto positivo en los estudiantes, sino que, además, en los docentes. Al respecto, según Pogré \& Lombardi (2004), esta promoción permitiría a los profesores repensar el proceso de enseñanza $y$, por tanto, reflexionar acerca de su propio actuar pedagógico en razón de resolver la tarea de colaborar en el desarrollo de comprensión en los estudiantes. Así mismo, los autores establecen que esta promoción conlleva un reconocimiento, por parte del profesor, de su importante función social.

\section{Evidencias de aplicación del modelo en Latinoamérica}

El modelo de enseñanza para la comprensión, desde su origen, fue orientado principalmente a ser aplicado en la educación escolar y es ahí, justamente, donde es posible encontrar mayor evidencia empírica en la actualidad respecto de su funcionamiento y efectividad (Salgado-García, 2012). En relación con su aplicación en instituciones de educación superior, existe evidencia en países latinoamericanos como Argentina, Costa Rica, Colombia, México. Sin embargo, dicha evidencia responde a la realización de experiencias pedagógicas particulares de grupos de investigación en el ámbito educativo más que a la aplicación del modelo como parte de la política curricular de una institución (Salgado-García, 2012).

Como parte de la evidencia de aplicación en instituciones de educación superior, se encuentra la experiencia de la Universidad Nacional de La Plata, en Argentina, donde se aplica de manera combinada Tecnologías de la Información y Comunicación (TIC), con un modelo de enseñanza para la comprensión. Dicha experiencia se da en el marco de un curso de Química básica implementado a distancia a un grupo de estudiantes de Ingeniería que realizaban la asignatura por segunda vez, pero donde la primera realización había sido en modalidad presencial. Los resultados apuntan a que todos los estudiantes mostraron avances significativos en la mayoría de los ítems trabajados, comparando el momento inicial de la asignatura, con el momento final. Se describen algunas dificultades en la forma de plantear las preguntas, así como la poca adherencia al formato de blog virtual con el cual se trabajó (Jubert et al., 2011).

Otra experiencia reportada se llevó a cabo en la Universidad Latinoamericana de Ciencia y Tecnología (institución de educación superior privada ubicada en Costa Rica). Dicha institución implementó el modelo de enseñanza para la comprensión en la totalidad de sus carreras. Luego de dos años de puesta en marcha, se aplicó un cuestionario para evaluar la efectividad del modelo según la percepción de los distintos actores del proceso de enseñanza-aprendizaje (estudiantes, graduados y personal académico). Los resultados muestran que los estudiantes y graduados, en un porcentaje mayor al 70\%, evaluaron el aprendizaje resultante, así como la exigencia de los proyectos aplicados (metodología 
de evaluación del modelo de enseñanza para la comprensión), como superior al de los exámenes tradicionales (metodología de evaluación de los modelos educativos tradicionales). Asimismo, su reporte de experiencia de las diversas estrategias de enseñanza-aprendizaje coherentes con el modelo de enseñanza para la comprensión, apuntan a que el modelo presenta un impacto en las prácticas educativas y en la experiencia de los estudiantes (Salgado-García, 2012).

\section{Reflexión final}

Con respecto a la problemática de la disociación entre el proceso de aprendizaje de saberes en el aula y la posterior dificultad en la aplicación de estos saberes en contextos prácticos, la solución no resulta sencilla, pues, como ya se describió previamente, el proceso de enseñanza-aprendizaje presenta un complejo andamiaje en el que participan múltiples factores de diversa naturaleza. Sin embargo, la explicación parece estar relacionada con la presencia o ausencia de procesos de enseñanza y aprendizaje comprensivos tanto en el aula como en el trabajo formativo realizado en contextos clínicos. La frecuente observación de una disociación entre el desempeño de los estudiantes en su trabajo teóricopráctico de aula y su posterior desempeño en un contexto clínico nos lleva a concluir que existe una profunda necesidad de promover el desarrollo de procesos comprensivos, mediante prácticas pedagógicas que estimulen el pensamiento analítico, reflexivo y, en definitiva, metacognitivo.

Nuestra experiencia nos muestra que el modelo centrado en competencia no permite completamente la transferencia o aplicación flexible de los conocimientos adquiridos en el aula hacia los campos clínicos. Si bien reconocemos que lo mencionado anteriormente puede estar relacionado con factores propios de las instituciones o de las carreras universitarias, más que con el modelo propiamente tal, pensamos que en este no se explicita la importancia de metodologías docentes tendientes al desarrollo de la comprensión profunda.

Por otra parte, si bien los resultados de investigaciones dan cuenta de la efectividad de la aplicación del modelo de enseñanza para la comprensión en instituciones latinoamericanas en comparación con modelos tradicionales de enseñanza aprendizaje, las experiencias reportadas no están exentas de complicaciones: por ejemplo, la resistencia a los cambios en las metodologías de evaluación desde pruebas escritas, propias de los modelos tradicionales del aprendizaje, a proyectos aplicados, propuestos por el modelo de enseñanza para la comprensión. Así mismo, como complicaciones se han reportado las experiencias educativas previas de los estudiantes. Estas experiencias, en general, van en la línea de acumulación de conceptos (Salgado-García, 2012). Cabe destacar que todas las experiencias descritas argumentan que la aplicación del modelo educativo no debe ser idéntico al original, sino que debe ajustarse al contexto cultural propio del país, así como de la institución en la cual se está implementando (Jubert et al., 2011; Salgado-García, 2012).

Según las limitaciones anteriores, se entiende que la aplicación del modelo de enseñanza para la comprensión en alguna institución de educación chilena requeriría fases de implementación, con un 
eje en la formación docente y en la transparencia de los cambios en los estudiantes. En esta línea, Salgado-García (2012) propone que deben existir 4 fases de implementación: a) Sensibilización inicial sobre el modelo, b) Proceso de actualización curricular, c) Construcción y evaluación permanente de los programas de los cursos, d) Capacitación al personal académico.

No cabe duda de que la implementación de un modelo educativo requiere de un profundo compromiso por parte de la comunidad educativa para su ejecución y permanente evaluación. Sin embargo, desde ya es posible la implementación de actividades que busquen desarrollar una comprensión profunda y verdadera de fenómenos de variada naturaleza en los estudiantes. En término de los saberes propios del campo de la fonoaudiología, es frecuente por ejemplo la enseñanza de teorías explicativas de cuadros clínicos o características diferenciadoras de estos mismos. En un modelo de enseñanza para la comprensión, la comprensión profunda de estos saberes se lograría gracias al análisis, al pensamiento crítico y la discusión de la información disponible, más que si se utilizan sólo estrategias de memorización. Dicha comprensión, probablemente, permitiría una transferencia más efectiva de aprendizajes desde el aula a contextos de ejercicio profesional, generando esto beneficios para todos los actores del ámbito fonoaudiológico, sean estos estudiantes, docentes, profesionales fonoaudiólogos y usuarios.

Otro punto relevante es el hecho de que si bien el modelo de enseñanza para la comprensión menciona la implementación de prácticas pedagógicas que estimulen el pensamiento analítico y reflexivo dentro del contexto de aula, es sabido que la formación en carreras de la salud es un proceso transversal desde las actividades en aula hasta la práctica clínica, es decir, el desarrollo del pensamiento metacognitivo debería continuar dentro de los campos clínicos, siendo este último un espacio propicio para lograr procesos comprensivos. Al respecto, Pérez-Peña (2008) y Safran \& Muran (2005) consideran que el docente clínico es un actor fundamental en el proceso de enseñanza-aprendizaje debido a que cumple el rol de mediador de este proceso en el ámbito clínico. Dicho ámbito, además, favorece la comprensión profunda de los saberes, dado que ofrece instancias distintas y diversas, para que los estudiantes apliquen de manera flexible los aprendizajes que han ido adquiriendo.

Probablemente el modelo centrado en competencias significa un acercamiento a los procesos comprensivos profundos en los estudiantes y el efecto en los procesos de transferencias podrán ser vistos con el tiempo. Sin embargo, pensamos que el conocer y reflexionar sobre el modelo de enseñanza para la comprensión puede significar un aporte para los procesos actuales de innovación curricular.

\section{Conclusiones}

1. El modelo de enseñanza para la comprensión promueve el desarrollo de procesos metacognitivos en los docentes y en los estudiantes, con el fin de llevar a estos últimos a procesos comprensivos profundos, favoreciendo la aplicación flexible de los contenidos trabajados en aula, en contextos diversos, tales como son los contextos 
clínicos en el ámbito de la fonoaudiología.

2. A partir de la propuesta inicial del Proyecto Zero, han surgido múltiples publicaciones sobre la aplicación del modelo de enseñanza para la comprensión, sin embargo la mayoría de los trabajos han estado referidos a la educación primaria y secundaria, y un escaso número de ellos se ha referido a la educación superior (Salgado-García, 2012). Por lo tanto, surge la necesidad de generar experiencias que se puedan evaluar, para aportar con datos empíricos a la discusión sobre la real efectividad de estos programas. Según Resnick (1999) la falencia de los programas existentes es justamente no contar con datos empíricos.

3. Una limitante a la aplicabilidad de este modelo en la educación superior está en el propio sistema educativo. En Chile, contamos con un sistema educativo basado en estándares, donde se explicitan las metas que deben alcanzar los actores educacionales y luego se evalúa el complimiento de estas. El riesgo de la estandarización en la educación es que se genere una visión simplificada de la misma y cayendo en el reduccionismo, es decir, se enseñe en post de lo que miden las pruebas estandarizadas (Casassus, 2010). En este sentido, se privilegia la eficacia y eficiencia en los tiempos y no la búsqueda de la comprensión.

4. En base a la reflexión planteada en este trabajo, se propone que el modelo centrado en competencias es constituye una manera de acercarse a los procesos comprensivos profundos en los estudiantes. Sin embargo, el modelo de enseñanza para la comprensión puede significar un aporte al entregar estrategias explícitas que permitan desarrollar procesos metacognitivos en los estudiantes, con el fin de favorecer la transferencia de aprendizajes desde el aula formal hacia la práctica clínica.

\section{Referencias}

Blythe, T. (1999). La enseñanza para la comprensión: guía para el docente. Buenos Aires: Paidós.

Casassus, J. (2010). Las Reformas Basadas en Estándares: un camino equivocado. En C. Bellei, D. Contreras, \& J. P. Valenzuela (Eds.), Ecos de la revolución pingüina: avances, debates y silencios en la reforma educacional (pp. 51-83). Santiago, Chile: Pehuén Editores S.A.

Díaz, A. (2006). El enfoque de competencias en la educación ¿una alternativa o un disfraz de cambio? Perfiles educativos, 28(111), 7-36.

Espinola, V., \& Claro, J. P. (2010). El sistema nacional de aseguramiento de la calidad: una reforma basada en estándares. En C. Bellei, D. Contreras, \& J. P. Valenzuela (Eds.), Ecos de la revolución pingüina: avances, debates y silencios en la reforma educacional (pp. 85-109). Santiago, Chile: Pehuén Editores S.A.

Gaete-Quezada, R. (2011). El juego de roles como estrategia de evaluación de aprendizajes universitarios. Educación y Educadores, 14(2), 289-307.

González, J., \& Wagenaar, R. (2003). Quality and European Programme Design in Higher Education. European Journal of Education, 38(3), 241-251. https://doi.org/10.1111/1467-3435.00144

Hawes, G., Rojas-Serey, A. M., Espinoza, M., Oyarzo, S., Castillo-Parra, S., Castillo, M., \& Romero, L. (2017). Desarrollo de una matriz conceptual para la innovación curricular en profesiones de la salud. Revista Médica de Chile, 145(9), 1193-1197. https://doi.org/10.4067/s003498872017000901193

Jonnaert, P., Masciotra, D., Barrette, J., Morel, D., \& Mane, Y. (2007). From Competence in the Curriculum to Competence in Action. PROSPECTS, 37(2), 187-203. https://doi.org/10.1007/s11125-007-9027-9

Jubert, A., Pogliani, C., Tocci, A. M., \& Vallejo, A. (2011). 
Enseñanza para la comprensión en un curso de química a distancia de nivel básico universitario. El blog como herramienta de trabajo. Avances en Ciencias e Ingeniería, 2(1), 97-105.

Klein, S. B. (2012). Learning: Principles and Applications (Edición: Sixth Edition). Thousand Oaks, Calif: SAGE Publications, Inc.

Le Boterf, G. (2004). Construire les compétences individuelles et collectives: La compétence n'est plus ce $q u^{\prime} e l l e ~ e ́ t a i t ~(3 e ~ e d$.$) . Paris: Éditions d'Organisation.$

Lefrancois, G. R. (2012). Theories of Human Learning: What the Professor Said (Edición: 6th ed.). Belmont, CA: CENGAGE Learning Custom Publishing.

Lund, N. (2010). Intelligence and Learning. New York: Macmillan International Higher Education.

Martínez, L., Cabezas, C., Labra, M., Hernández, R., Martínez, L. M., Cerutti, M., \& Malebrán, C. (2006). La logopedia en Iberoamérica. En Actas del XXV Congreso de Logopedia, Foniatría y Audiología (p. 14). Granada, España. Recuperado http://www.sld.cu/galerias/pdf/sitios/rehabilitacionlogo/historia_en_iberoamerica.pdf

McTighe, J., \& Self, E. (2014). Teaching for understanding: A meaningful education for 21st century learners [open access]. Teachers Matter, 24, 15-17.

Pastorino, E., \& Doyle-Portillo, S. (2016). What Is Psychology?: Foundations, Applications, and Integration (Edición: 3rd ed.). Boston MA: CENGAGE Learning Custom Publishing.

Pérez-Peña, F. (2008). El papel del profesor de práctica clínica. Educación Médica, 11, 37-42.

Perkins, D. (1993). Teaching for Understanding. American Educator: The Professional Journal of the American Federation of Teachers, 17(3), 28-35.

Perrenoud, P. (2004, junio). L'université entre transmission de savoirs et développement de compétences. Presentado en Congreso de la enseñanza universitaria y de la innovación, Girona, España. Recuperado de https://www.unige.ch/fapse/SSE/teachers/perrenoud/php _main/php_2004/2004_07.html
Perrenoud, P. (2007). Desarrollar la práctica reflexiva en el oficio de enseñar. México: Editorial Graó. Recuperado de https://www.noveduc.com/l/desarrollar-la-practicareflexiva-en-el-oficio-de-ensenar/1331/9789875384941

Pogré, P., \& Lombardi, G. (2004). Escuelas que enseñan a pensar: enseñanza para la comprensión, un marco teórico para la acción. Buenos Aires: Papers Editores.

Resnick, L. (1999). La educación y el aprendizaje del pensamiento. Buenos Aires: Aique.

Resnick, L., \& Collins, A. (1996). Cognición y aprendizaje. Anuario de Psicología, 69, 189-197.

Rodríguez, H. (2007). El paradigma de las competencias hacia la educación superior. Revista de la Facultad de Ciencias Económicas, 15, 145-165.

Safran, J. D., \& Muran, J. C. (2005). La alianza terapéutica: una guía para el tratamiento relacional. Bilbao: Desclée de Brouwer. Recuperado de https://dialnet.unirioja.es/servlet/libro?codigo=251656

Salgado-García, E. (2012). Enseñanza para la comprensión en la educación superior: la experiencia de una universidad costarricense. Revista iberoamericana de educación superior, 3(8), 34-50.

Stone, M. (1999). La enseñanza para la comprensión: vinculación entre la investigación y la práctica. Buenos Aires: Paidós.

Troncoso, K., \& Hawes, G. (2007). Esquema general para los procesos de transformación curricular en el marco de las profesiones universitarias. Santiago: Dirección de Pregrado, Vicerrectoría de Asuntos Académicos, Universidad de Chile.

Van Berkel, H., Scherpbier, A., Hillen, H., \& van der Vleuten, C. (2010). Lessons from Problem-based Learning (Edición: 1). Oxford: Oxford University Press.

Wray, D., \& Lewis, M. (1997). Extending Literacy: Children Reading and Writing Non-fiction. London: Routledge. 